\title{
Early use of an implantable diaphragm pacing stimulator for a child with severe acute flaccid myelitis-a case report
}

\author{
Travis L. Edmiston ${ }^{1,2} \cdot$ Mathew J. Elrick $\mathbb{1}^{3} \cdot$ Mark L. Kovler $^{4} \cdot$ Eric B. Jelin ${ }^{4} \cdot$ Raymond P. Onders ${ }^{5} \cdot$ \\ Cristina L. Sadowsky $\mathbb{D}^{1,2}$
}

Received: 9 May 2019 / Revised: 24 June 2019 / Accepted: 24 June 2019

(C) International Spinal Cord Society 2019

\begin{abstract}
Introduction Acute Flaccid Myelitis (AFM) is a recently recognized, polio-like illness of children that can be functionally devastating. Severe cases can lead to ventilatory failure. Incomplete phrenic nerve injuries in other populations has been shown to respond to diaphragmatic stimulation. We therefore proposed an early assessment for incomplete denervation by laparoscopic direct stimulation of the diaphragm and placement of a diaphragmatic pacing system to enhance diaphragm function.

Case presentation A 3 year-old girl presented with AFM with clinically and electrodiagnostically severe involvement of all four limbs and muscles of respiration. Direct stimulation of the diaphragm demonstrated contraction and a diaphragmatic stimulator was placed at 3 weeks post presentation. The patient was immediately able to tolerate short bouts of reduced ventilation settings. Electromyography via the pacing wires demonstrated intact motor units consistent with partial denervation/reinnervation in the left hemidiaphragm, and no motor units in the right hemidiaphragm. At three months, she tolerated $6 \mathrm{~h}$ of pacing on pressure support setting. At 5 months she demonstrated larger tidal volumes with active pacing than without. Discussion In our experience, AFM patients who require chronic ventilator support are rarely able to be weaned. Despite clinical and surface electrodiagnostic evidence of complete phrenic nerve involvement, the patient's diaphragm responded to direct stimulation. The patient preferred pacing over non-pacing times and showed improved ventilatory ability with pacing as opposed to without, though remains ventilator-dependent. These findings support augmentation of diaphragm function and possible enhanced recovery of spontaneous function.
\end{abstract}

\section{Introduction}

Acute Flaccid Myelitis (AFM) is a recently recognized and increasingly prevalent polio-like illness resulting in flaccid

Travis L. Edmiston

Edmiston@kennedykrieger.org

1 International Center for Spinal Cord Injury, Kennedy Krieger Institute, Baltimore, MD, USA

2 Department of Physical Medicine and Rehabilitation, Johns Hopkins School of Medicine, Baltimore, MD, USA

3 Department of Neurology, Johns Hopkins School of Medicine, Baltimore, MD, USA

4 Department of Pediatric Surgery, Johns Hopkins School of Medicine, Baltimore, MD, USA

5 Department of General Surgery, University Hospitals Cleveland Medical Center, Cleveland, OH, USA paralysis. AFM generally affects young children through a presumed neuroinfectious or neuroinflammatory process of the spinal cord grey matter following an otherwise unremarkable viral illness. A preference for anterior gray matter results in rapid lower motor neuron weakness in the limbs, cranial nerves and/or muscles of respiration. The completeness of the paralysis and subsequent recovery are extremely variable and appear to be best predicted by the severity of involvement at the disease peak [1]. Many children are successfully weaned from ventilator dependence, but some remain on ventilatory support chronically following their initial presentation. As of May 2019, the Centers for Disease Control and Prevention have confirmed 264 cases for the 2018 season [2].

The minimally invasive diaphragmatic pacing system (DPS) has been shown to reduce ventilator dependence in pediatric populations with intact phrenic innervation, such as following injury to the cervical spinal cord or the PreBotzinger complex of the brainstem [3,4]. DPS has been 
shown to help recover phrenic nerve function and maintain diaphragm muscle mass especially when begun early [5]. If there is complete loss of the anterior horn cells or complete transection of the phrenic nerve, then the phrenic nerve undergoes Wallerian degeneration and becomes non-stimulatable; the diaphragm then atrophies and undergoes fibrotic replacement. It stands to reason that a patient with AFM and partial phrenic denervation may benefit from diaphragmatic pacer placement to augment diaphragm function and thereby limit ventilator dependence. Anecdotally reported, AFM patients with respiratory insufficiency have not benefited from DPS placed at later timepoints. There is limited experience with two such chronic ventilator-dependent children who underwent DPS implantation 6 months or more after the onset of neurologic deficits and who were unsuccessful in weaning. Early use of DPS after traumatic SCI has been shown to have significant benefit without long-term adverse events [6, 7]. We reasoned that early placement of DPS in AFM may promote axonal sprouting and reinnervation and/or prevent diaphragmatic atrophy, and may therefore have a higher likelihood of success. We hereby report the first attempt at DPS placement in acute phase AFM.

\section{Case presentation}

The patient is a 3-year-old previously healthy and neurodevelopmentally appropriate girl who initially presented with a viral illness four days prior to onset of weakness. Over the next two days she developed neck and left arm pain, then inability to ambulate, followed by inability to sit unsupported. On admission, her lower extremities were flaccid with $0 /$ 5 strength, absent reflexes, and normal sensation. Over the next $24 \mathrm{~h}$, she developed progressive weakness of the upper extremities and neck, lost the ability to speak, and was intubated for impending respiratory failure.

Magnetic resonance imaging (MRI) showed T2 hyperintensity of the central grey matter from the cervicomedullary junction to the conus medullaris and subtle increased signal in the dorsal pons and medulla. Electromyography and nerve conduction studies (EMG/ NCS) of her extremities performed 7 days after weakness onset demonstrated absent compound muscle action potentials (CMAP), marked fibrillation potentials and positive sharp waves, and no voluntary motor unit potentials (MUP). The diagnosis of Acute Flaccid Myelitis was made according to the Centers for Disease Control and Prevention case definition on the basis of acute limb weakness with a grey matter predominant spinal cord lesion on MRI, and further supported by CSF pleocytosis, identification of the EV-68 virus from nasopharyngeal swab, motor neuronopathy, and exclusion of alternative diagnoses [8].

Soon after intubation, discussions began about the role of intraoperative evaluation of the diaphragm by electrical stimulation and possible implantation of a diaphragmatic stimulator. Intraoperative stimulation has demonstrated response and benefit from placement even when surface electrodiagnostics have not shown diaphragmatic innervation.(4) She was taken to the operating room on hospital day 21 for laparoscopic diaphragm mapping and pacer (Fig. 1) (NeuRx, Synapse Biomedical) implantation as previously described in children with slight modification [9]. The posterior left diaphragm was responsive and electrodes were placed at the identified motor points. The right diaphragm was less responsive; however, using train stimulation it did contract diffusely and a motor point was identified.

Immediately following placement, she was able to tolerate short bouts of DPS-supported continuous positive airway pressure and pressure support (CPAP/PS) breathing. On hospital day 28 she was transferred to inpatient rehabilitation tolerating two sessions of $30 \mathrm{~min}$ of DPS-driven breathing. Chest X-rays were obtained to monitor for any increasing atelectasis with increasing duration of pacing. At $6 \mathrm{~h}$ of continuous pacing each day, it was felt that a balance of benefit from pacing and risk of atelectasis was reached (Table 1).
Fig. 1 Diaphragm Pacing System. Left panel: External stimulator (a), patient cable (b), and connector with skin badge (c). Right panel: Pacing system connected to the exiting portion of the electrode wires (d)
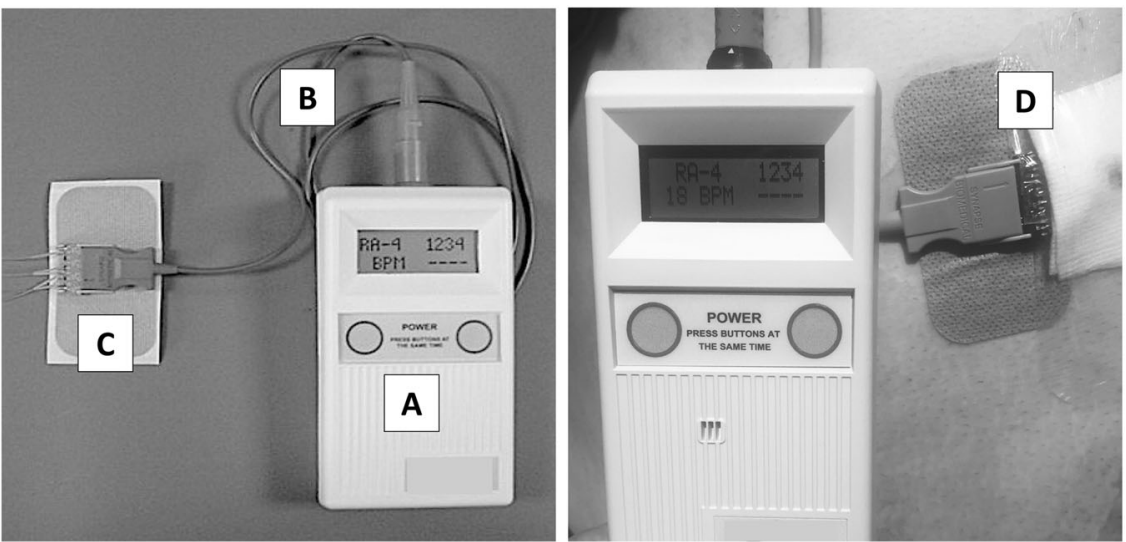


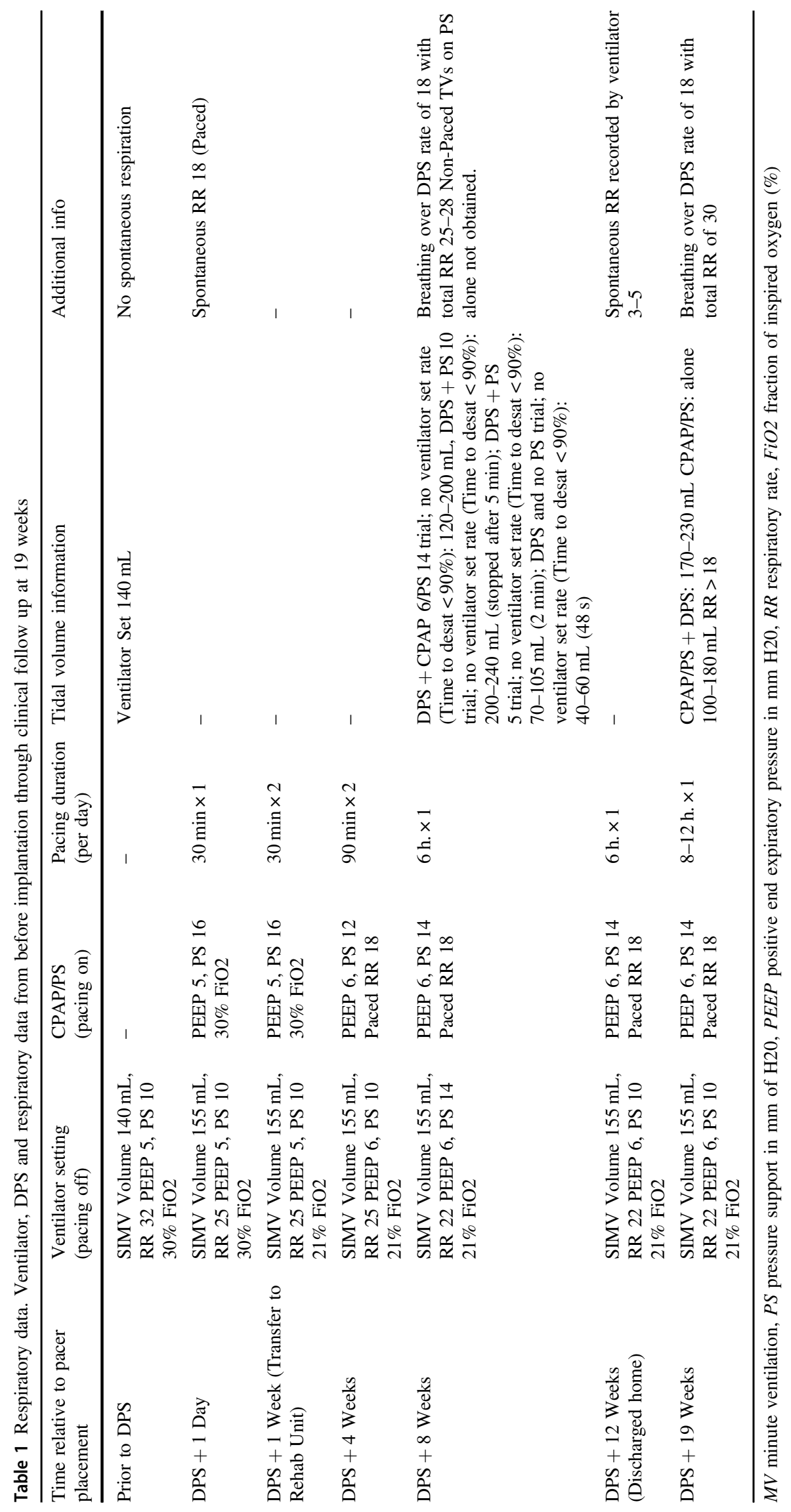


During her rehabilitation course, she underwent diaphragm ultrasound evaluation which demonstrated significantly better left diaphragmatic excursion with DPS or spontaneous respirations alone over ventilator support with or without active pacing. The right hemidiaphragm showed fluttering without excursion. She was also assessed for tidal volumes (TV) and time to under $90 \%$ oxygen desaturation under different PS conditions while being paced. The patient was able to maintain an age-appropriate TV with a PS of $10 \mathrm{~mm} \mathrm{H} 2 \mathrm{O}$ without desaturation, a TV of $70-105 \mathrm{~mL}$ for $2 \mathrm{~min}$ at a PS of $5 \mathrm{~mm} \mathrm{H} 2 \mathrm{O}$ and finally a TV of $40-60 \mathrm{~mL}$ for $48 \mathrm{~s}$ without PS (Table 1).

At two months post-presentation, CMAP of bilateral phrenic nerves was still not detectable by surface electrodes. A continuous diaphragmatic EMG was conducted using the pacing wires as previously described [5] which allowed for evaluation of epochs of respiration with cardiac activity in the background. An episode of apnea was captured (Fig. 2).
With the DPS off, the recording of left diaphragm showed reduced recruitment of MUPs of normal amplitude and mildly prolonged duration, supportive of denervation and reinnervation. On the right, there were no motor units, consistent with complete denervation (Fig. 3).

At 19 weeks post DPS placement, EMG through DPS showed multiple motor units with amplitude of approximately $1000 \mu \mathrm{V}$. Tidal volumes were evaluated under equivalent ventilator settings with and without active pacing. She achieved TV of 100-180 mL on CPAP/PS with a volitional rate greater than the backup rate of 18 and a larger TV of 170-230 mL while supported by DPS.

Her rehabilitation course was complicated by an unrelated home ventilator malfunction requiring intensive care readmission for replacement. She had no other respiratoryrelated complications. At the time of discharge, she was able to partially activate neck rotation as well as partially flex her right fifth digit and left great toe.

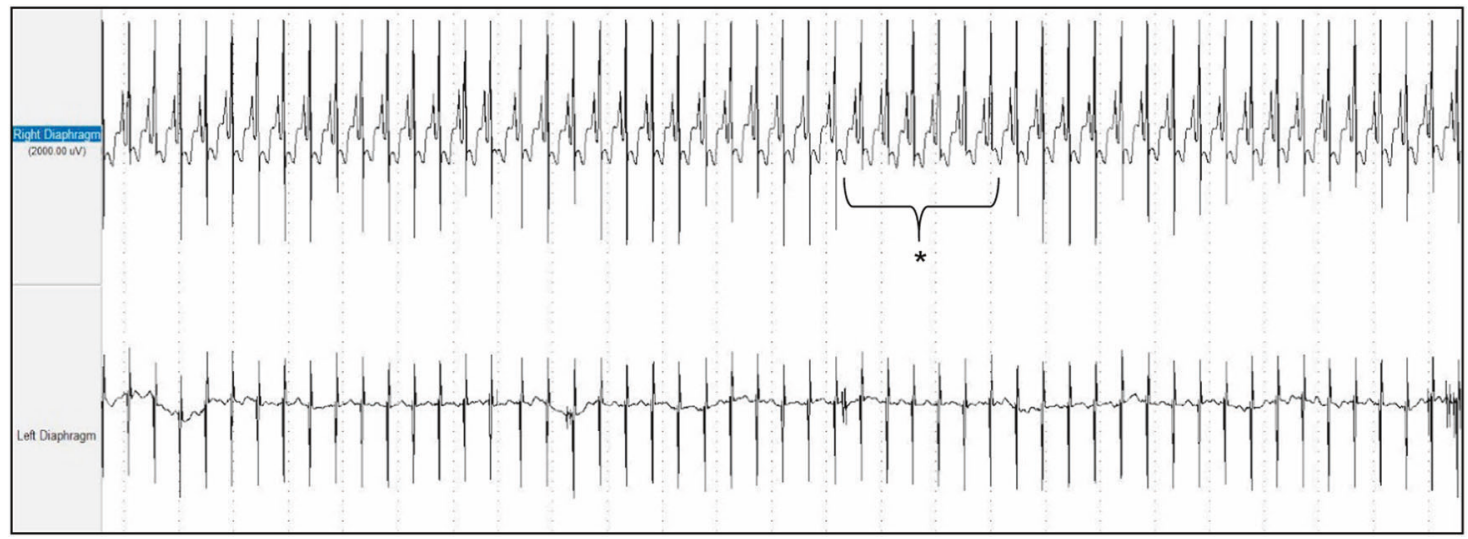

Fig. 2 Continuous diaphragm EMG showing background cardiac activity. The left diaphragm (upper trace with apnea event noted*) and right diaphragm (lower trace with absent motor units)

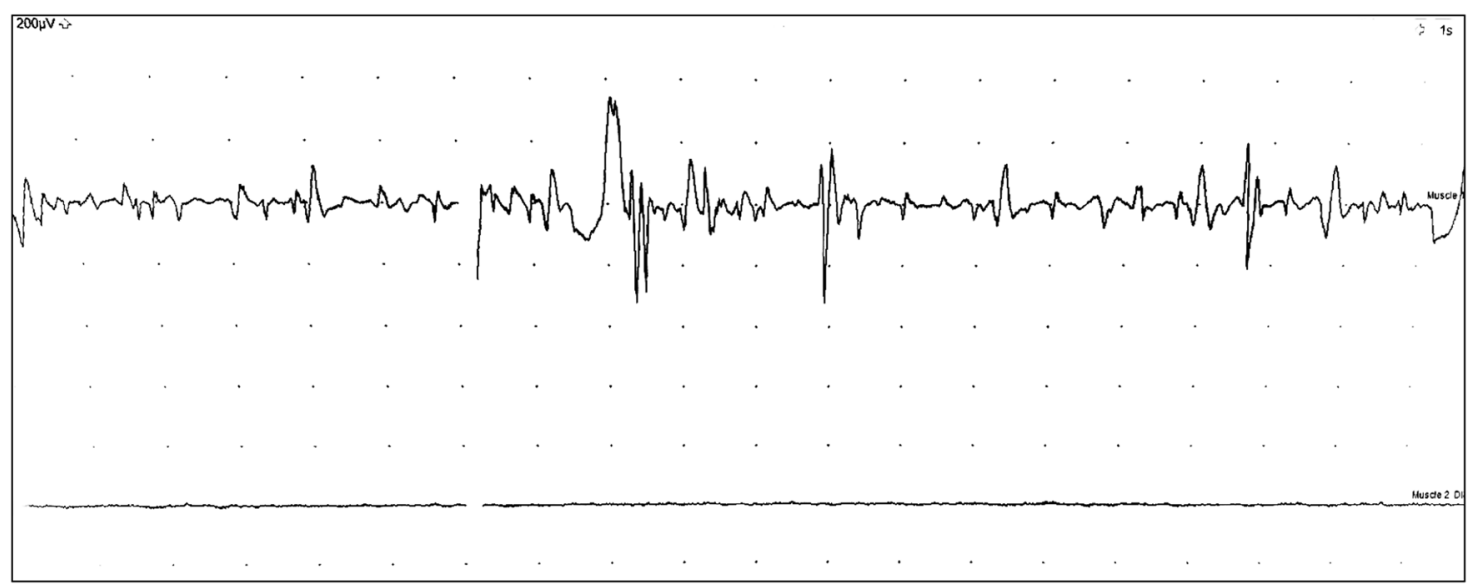

Fig. 3 Electromyography of the left (upper trace) and right (lower trace) hemidiaphragms under spontaneous respiration. Gain $200 \mu$ V/div, sweep $50 \mathrm{~ms} / \mathrm{div}$ 


\section{Discussion}

AFM can be functionally devastating. In our more than 10 years of retrospective experience, AFM patients who require chronic ventilator support are rarely able to be weaned. We proposed an early attempt at DPS in the hopes that pacer placement would maintain active diaphragm function and allow for reduced passive ventilator dependence. Critical to the efficacy of this approach in our patient was the identification of partially intact innervation of the left hemidiaphragm allowing for effective stimulation of diaphragmatic contraction by the DPS. This was detectable only by intraoperative stimulation. Surface electrodes were unable to detect a phrenic CMAP and clinical evaluation of diaphragm pre-operatively suggested complete paralysis. This echoes the experience in the spinal cord injury population, where phrenic nerve testing by surface EMG has been felt by some surgical teams to be less sensitive than direct intraoperative visualization [4].

To our knowledge, other individuals with AFM have undergone diaphragmatic pacer placement; however, this was in the chronic setting, with minimal subsequent reduction in ventilator support. In our clinical observations with AFM, muscle atrophy is rapid. We suspect that failure of DPS in these patients may be due to marked atrophy or complete denervation of diaphragmatic muscle prior to pacer placement. Although electrical stimulation is insufficient to provide trophic support to muscle, experimental data suggests that electrical stimulation following nerve injury, particularly at early time points, promotes sprouting and reinnervation [10]. Importantly, axonal sprouting is thought to be the primary means of functional recovery in AFM. Therefore, our patient's improved respiratory function following DPS implantation may be due to stimulation of axonal sprouting and reinnervation and/or enhancement of phrenic nerve activation beyond that which can be generated voluntarily.

An alternative explanation for the patient's decreased ventilator dependence is simply spontaneous recovery of voluntary motor function to the partially denervated left phrenic nerve. However, two lines of evidence support a direct role for DPS in improving her respiratory function. First, she gained the ability to tolerate short bouts of reduced ventilatory support immediately following DPS placement. Second, her TVs were larger with the DPS turned on than with ventilator support alone.

An episode of apnea was seen on continuous diaphragm EMG (Fig. 2). This could be explained by her known brainstem involvement further impairing ventilator weaning. The role of brain stem-mediated apnea in AFM has not been investigated; however, the use of diaphragmatic stimulation may prevent predictable diaphragmatic muscle atrophy.
This patient was not fully weaned from ventilator dependence. She did, however, show pacing over full ventilator support and had larger TV while being paced. She continues to work towards ventilator independence with the daily use of DPS at home without complication. Many children become successful at hemidiaphragm ventilation as they age [11, 12]. Additionally, early implantation to maintain diaphragm muscle allows for the possibility of intercostal nerve transfer if there is no recovery of the right hemidiaphragm as has been done in the traumatic pediatric SCI population [13]. It is our hope that through skeletal maturation, axonal sprouting, and active diaphragmatic pacing, this patient could potentially achieve some level of ventilator independence.

In conclusion, early use of DPS in AFM allows for both diagnostic evaluation of the diaphragm and the potential to improve respiratory function for possible future ventilator weaning. Continued evaluation of this and other interventions targeting functional recovery is warranted in this recently-recognized disease.

Acknowledgements Janet M. Dean, MS, RN, CRRN, CRNP for expertise and clinical management. Suzanne Venoski Rybczynski, MD for expertise and clinical management. Michelle Melicosta, MD, $\mathrm{MPH}$, FAAP for expertise and clinical management. Thomas Owen Crawford, M.D. for expertise and electrodiagnostics

\section{Compliance with ethical standards}

Conflict of interest The authors declare that they have no conflict of interest.

Publisher's note: Springer Nature remains neutral with regard to jurisdictional claims in published maps and institutional affiliations.

\section{References}

1. Gordon-Lipkin E, Muñoz LS, Klein JL, Dean J, Izbudak I, Pardo CA. Comparative quantitative clinical, neuroimaging, and functional profiles in children with acute flaccid myelitis at acute and convalescent stages of disease. Dev Med Child Neurol. 2018. https://doi.org/10.1111/dmcn.14030.

2. CDC. Acute Flaccid Myelitis I AFM Surveillance I. 2019. https://www.cdc.gov/acute-flaccid-myelitis/afm-surveillance.html.

3. Onders RP, Elmo MJ, Ignagni AR. Diaphragm pacing stimulation system for tetraplegia in individuals injured during childhood or adolescence. J Spinal Cord Med. 2007;30(Suppl 1):S25-9.

4. Dean JM, Onders RP, Elmo MJ. Diaphragm pacers in pediatric patients with cervical spinal cord injury: a review and implications for inpatient rehabilitation. Curr Phys Med Rehabil Rep 2018;6:257-63.

5. Onders RP, Elmo M, Kaplan C, Katirji B, Schilz R. Extended use of diaphragm pacing in patients with unilateral or bilateral diaphragm dysfunction: a new therapeutic option. Surgery. 2014;156:776-84.

6. Onders RP, Elmo M, Kaplan C, Schilz R, Katirji B, Tinkoff G. Long-term experience with diaphragm pacing for traumatic spinal 
cord injury: early implantation should be considered. Surgery. 2018;164:705-11.

7. Kerwin AJ, Yorkgitis BK, Ebler DJ, Madbak FG, Hsu AT, Crandall ML. Use of diaphragm pacing in the management of acute cervical spinal cord injury. J Trauma Acute Care Surg. 2018;85:928-31.

8. Ayscue P, Van Haren K, Sheriff H, Waubant E, Waldron P, Yagi $\mathrm{S}$, et al. Acute flaccid paralysis with anterior myelitis-California, June 2012-June 2014. MMWR Morb Mortal Wkly Rep. 2014;63:903-6. 10

9. Onders RP, Ponsky TA, Elmo M, Lidsky K, Barksdale E. First reported experience with intramuscular diaphragm pacing in replacing positive pressure mechanical ventilators in children. $\mathbf{J}$ Pedia Surg. 2011;46:72-6.
10. Al-Majed AA, Neumann CM, Brushart TM, Gordon T. Brief electrical stimulation promotes the speed and accuracy of motor axonal regeneration. J Neurosci. 2000;20:2602-8.

11. Langer JC, Filler RM, Coles J, Edmonds JF. Plication of the diaphragm for infants and young children with phrenic nerve palsy. J Pedia Surg. 1988;23:749-51.

12. Baker CJ, Boulom V, Reemtsen BL, Rollins RC, Starnes VA, Wells WJ. Hemidiaphragm plication after repair of congenital heart defects in children: quantitative return of diaphragm function over time. J Thorac Cardiovasc Surg. 2008;135:56-61.

13. Latreille J, Lindholm EB, Zlotolow DA, Grewal H. Thoracoscopic intercostal to phrenic nerve transfer for diaphragmatic reanimation in a child with tetraplegia. J Spinal Cord Med. 2019. https://doi. org/10.1080/10790268.2019.1585706 [Epub ahead of print]. 